\title{
A Proposed Road Map for the Ethical Evaluation of Sham (Placebo) Surgery
}

\author{
Vittoradolfo Tambone, MD, PhD, ${ }^{*}$ Dario Sacchini, $M D, P h D, M A(P h i l), \dagger$ \\ Antonio G. Spagnolo, MD, BA (Phil), $\dagger$ Rosa Menga, MD, $\ddagger$ Giovanna Ricci, PhD, $\S$ Roberto Valenti, MA, ${ }^{* *}$ \\ Massimiliano Andrea Vitali, MD, ${ }^{*}$ and Massimo Ciccozzi, MA*
}

\begin{abstract}
Objective: The study proposes a possible roadmap for the ethical assessment of sham surgery clinical trials (CTs), focusing on methodological aspects, as a result of the lack of this type of practical tool in the literature/practice.

Background: Surgical procedures are frequently conducted without closely controlled studies. For this reason, these procedures are less rigorous than those for drug/device clinical trials. The aim of a sham (placebo) surgery CT is to carry out a surgical CT with a legitimate control group. The use of sham surgery is controversial from an ethical point of view.

Methods: This evaluation system is set up according to ICH/GCP, World Medical Association Declaration of Helsinki, CONSORT 2010 standards. The proposed roadmap is based on the following 4 steps/levels: safety/clinical indications; adequacy of trial methodology/design adopted for a sham surgery CT; specific informed consent, and economic issues.

Results: A flowchart is proposed which can be used at two levels: as a basic guideline for the design of a surgical protocol representing a benchmark level of care; and a multiaxial assessment considering the first two sources of morality of human acts according to Aristotelian ethics: the object of the act (step 1) and some of its circumstances (steps 2-4).

Conclusions: The use of a placebo and of double-blind control groups in surgery CTs would improves the quality of results, providing that an accurate ethical assessment procedure is in place, firstly to ensure patient safety and secondly to prevent abuses/procedural biases. Future testing of the proposed flowchart is outlined.
\end{abstract}

Keywords: assessment, clinical experimentation, ethics, sham surgery

(Ann Surg 2017;265:658-661)

C urrent surgical procedures are frequently based on uncontrolled studies: few controlled randomized surgical clinical trials (RCTs) are reported in the literature. Therefore, these procedures are less rigorous than those for drugs and/or devices. The standard of assessment for surgery is usually lower, because of the greater complexity of conducting surgical RCTs in terms of both scientific validity and ethical soundness. A double-blind/placebo-controlled RCT represents a benchmark standard in the evaluation of new

From the *Institute of Philosophy of Scientific and Technological Activity, University Campus Bio-Medico of Rome, Italy; Via Alvaro del Portillo 21, Rome, Italy; †Institute of Bioethics and Medical Humanities, "A. Gemelli", School of Medicine and Surgery, Università Cattolica del Sacro Cuore; Largo Francesco Vito 1, Rome, Italy; $\ddagger$ Faculty of Medicine and Surgery, University Campus Bio-Medico of Rome, Italy; Via Alvaro del Portillo 21, Rome, Italy; $\S$ School of Law, University of Camerino; Piazza Cavour 19, Camerino (MC), Italy; **Department of Engineering, University Campus Bio-Medico of Rome, Italy; Via Alvaro del Portillo 21, Rome, Italy; and ||Istituto Superiore di Sanità, Rome, Italy.

The authors declare that there are no conflicts of interest.

Reprints: Dario Sacchini, MD, PhD, MA (Phil), Institute of Bioethics and Medical Humanities, "A. Gemelli" School of Medicine, Università Cattolica del Sacro Cuore, Largo F. Vito 1, 00168 Rome, Italy. E-mail: dario.sacchini@ unicatt.it. Copyright (C) 2016 Wolters Kluwer Health, Inc. All rights reserved.

ISSN: 0003-4932/16/26504-0658

DOI: $10.1097 /$ SLA.0000000000002007 procedures/drugs, ensuring avoidance of biases while monitoring the placebo effect. As regards clinical research, it is clear that the risk for the placebo group during surgical trials is higher than in pharmacological trials.

Sham surgery is "a treatment or procedure that is performed as a control and that is similar to, but omits a key therapeutic element of, the treatment or procedure under investigation." ${ }^{1}$ The aim is to carry out a surgical RCT with a control group, thereby permitting comparison of a surgical technique with a sham (placebo) procedure and thus achieving a double-blind/placebo-controlled RCT.

According to Wartolowska et al, 53 clinical trials have been performed with a sham control group. The authors conclude that "the results provide evidence against continued use of the investigated surgical procedures. Without well-designed placebo controlled trials of surgery, ineffective treatment may continue unchallenged."2 Despite this, "surgery of any form, including placebo surgery, is associated with some level of risk, whereas a placebo tablet or drug is not (...). Therefore, the balance between risks and benefits in placebo surgically controlled RCTs is different from that in drug trials." 2 Indeed, the use of sham surgery is much debated from the ethical point of view, ${ }^{3-8}$ particularly regarding ethical issues, deception, and informed consent. According to Tenery et al, ${ }^{6}$ "In addition however, the use of surgical placebo controls requires a careful assessment of the specific scientific benefits, and also surgical risks, such as anesthesia or infection, which should be as low as possible."

Already in 1961, Beecher ${ }^{4}$ stated that researchers should investigate the extent of the placebo effect so that dangerous surgical procedures that were no more effective than placebos would not be performed. A report on internal mammary artery ligation in $1950 \mathrm{~s}^{3,6}$ for patients with myocardial ischemia proves the point. Consequently, strict requirements such as those proposed by the Council on Ethical and Judicial Affairs of the American Medical Association (2002) are needed for ensuring the ethical acceptability of sham surgery. 6

Given the inherent conflict between observing the highest standards in both research design and ethics, our opinion is that neither should prevail, but that the highest level of research design should be combined with the highest ethical standards. In the meantime, we believe that the debate on the use of sham surgery is overly focused on specialist cases, whereas we need an affordable, and hopefully shared, framework for ethical assessment. Because, in general, ${ }^{5-7}$ the literature includes few attempts at producing requirements for ethically sound sham surgery, ${ }^{7}$ despite several surgical trials including sham surgery in their design are registered [648 ongoing or completed trials are listed in "clinicaltrial.gov" website (accessed May 26, 2016)], and also considering the not yet fully resolved ethical reflections on it, we believe it may be useful to propose a methodological road map for more detailed and accurate ethical assessment. This tool can be useful for both Independent Ethics Committees/Institutional Review Boards (IEC/IRB) and single researchers. 


\section{A ROAD MAP FOR THE ETHICAL EVALUATION OF SHAM SURGERY}

This evaluation system is based on the following 4 levels: safety and clinical indications; methodology; informed consent, and economic issues (Fig. 1).

\section{Safety and Clinical Indications (First Level of Evaluation)}

According to the standards defined in International Conference on Harmonisation/Good Clinical Practice (ICH/GCP) Guidelines, the World Medical Association (WMA) Declaration of
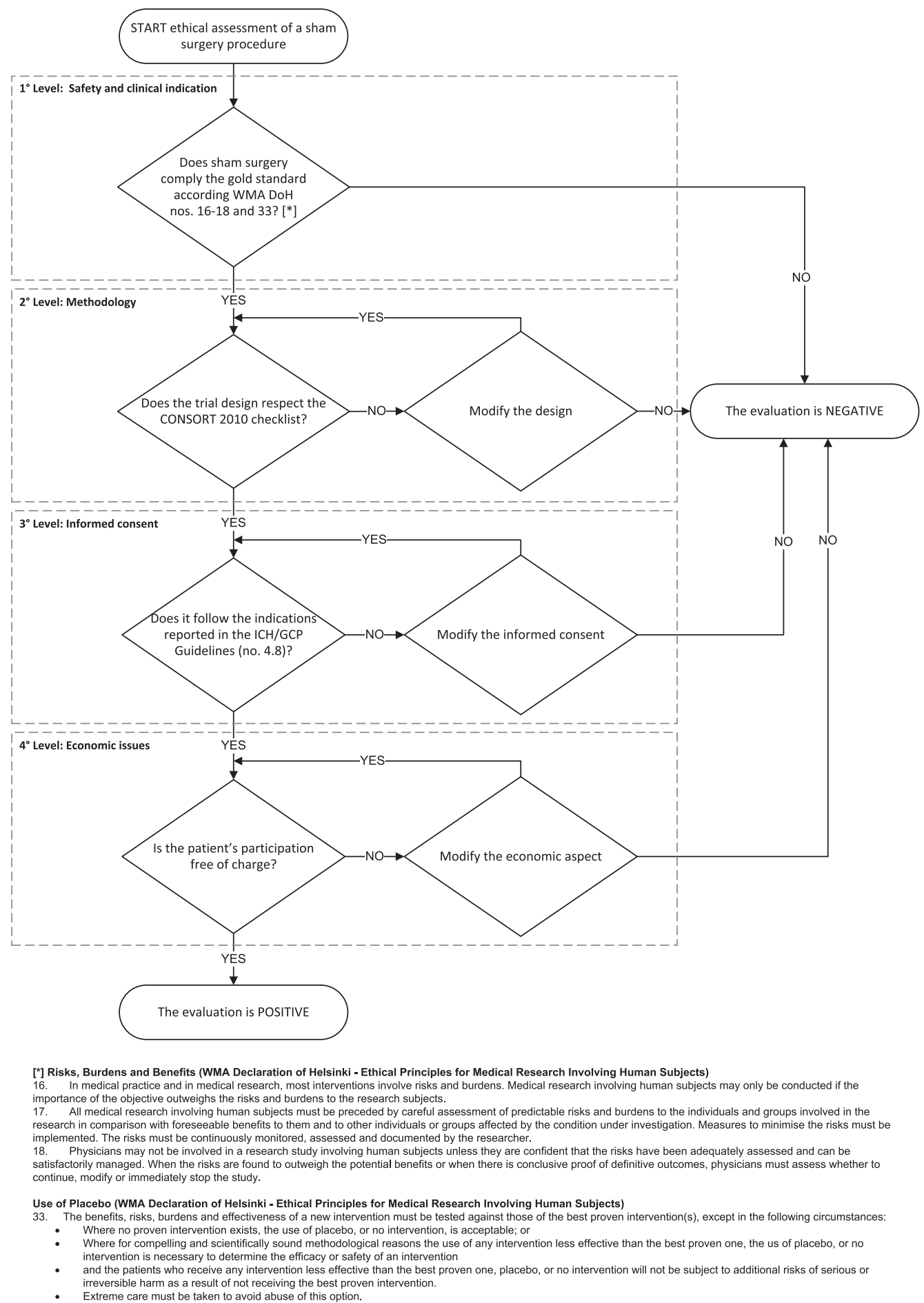

FIGURE 1. Flowchart of the ethical assessment of a sham surgery procedure. 
Helsinki (WMA DoH) and new European Union (EU) Regulation No 536/2014, ${ }^{9-11}$ all patients enrolled on a clinical trial must be provided with the best available treatment and with suitable insurance to cover any possible damage that could be suffered. Clearly, when a sham surgery clinical trial is submitted to an IEC/IRB, the principal investigator is charged to show evidence that there are no alternative surgical therapies.

For these reasons, the first level of evaluation involves answering the following questions:

- Is sham surgery the best available treatment in this case?

- If yes, is robust evidence concerning the treatment protocol available?

- Is appropriate insurance provided?

Apart from these three points which derive from the cited documents, the only addendum that we suggest for this first step is an assessment of the patient's psychological/psychiatric state, and his/her understanding and motivation to participate in a placebo clinical trial because of its relevance in a study regarding the placebo effect, thereby avoiding the "therapeutic misconception."

The first level of ethical evaluation should comply with the standards checklists of GCP, the WMA-DoH, and new EU Regulation No 536/2014, and address the questions above.

This first level should be satisfied before moving on to the next.

\section{Methodology (Second Level of Evaluation)}

The introduction of sham surgery is justified by a desire for the highest standard of research reporting, in accordance with Consolidated Standards of Reporting Trials (CONSORT), ${ }^{12}$ the reference standard. The CONSORT 2010 checklist thus represents the second step of the evaluation. For our evaluation, the following points are considered specifically, with the addition of an addendum:

- "Criteria of eligibility" must be chosen to ensure avoidance of any possible additional risk as regards the standard intervention; patients must not have undergone previous surgical intervention; inclusion and exclusion criteria must be homogeneous.

- The "intervention" must be described in adequate detail to allow replication (addendum 1); this description must be made without falsifying any data; postsurgical double-blindness has to be assured, with the patient's informed consent. On the other hand, when the patient safety (eg, a new diagnostic/therapeutic intervention) requires the patient access to medical record, we must to break the double blindness and the patient get out from the trial.

- "Outcome measures" must be detailed. Moreover (addendum 2), if applicable, which patients were assigned "blind" to intervention, and how, should be specified. If relevant, description of the similarities between interventions should be provided.

- "Trial limitations," addressing sources of potential bias, imprecision, and, if relevant, multiplicity of analyses, should be described. Moreover (addendum 3), in this regard, confounding identification is relevant.

- "Generalizability" (external validity, applicability) of the trial findings must be ensured.

This second level should be observed before moving on to the third step of evaluation.

\section{Informed Consent (Third Level of Evaluation)}

Informed consent should be obtained in accordance with the ICH/GCP Guidelines (no. 4.8), ${ }^{9}$ including the provision of clear and accurate information to all involved subjects. Moreover, the patients should have no knowledge about the type of intervention sustained until the end of the follow-up; therefore the patients are not allowed to read their own medical records.

\section{Economic Issues (Fourth Level of Evaluation)}

On one hand, a patient's participation in a sham surgery trial must be free of charge, including any economic burden(s) related to complications arising from the sham operation. On the other hand, it is necessary to guarantee the postsurgical double blindness of the trial. For ensuring the double blindness, one of the necessary elements will be the equal economic treatment of the two arms (free of charge), as usual in all clinical trials.

\section{Final Analysis}

As regards the first level (safety and clinical indications), all the issues have to be addressed. The final result can be positive or negative. Only if the answer to the first level requirements is positive can we move on to the second level. If the score is negative, the general evaluation will be negative regardless of other issues. Therefore, for carrying out the trial, modifications are required in accordance with international standards. The same procedure should be observed for the second level. The evaluation of the third level (informed consent) relies on the following: an informed consent form, fulfilling GCP standards; no possibility that the patient enrolled in the sham surgery trial might consult his/her medical record until the end of the follow-up of the trial. Only in the case of a positive answer it is possible to move to the next level. The fourth level (economic) of assessment is satisfied if financial burdens for the patient and different treatments are excluded. The final result of the ethical evaluation is positive if the results are positive for all the four levels, or negative if the analysis is interrupted due to negative results at any level (this level is to be declared).

\section{RECOMMENDATIONS ABOUT THE UTILIZATION OF THE ROAD MAP}

For assessing the adherence to the indications provided by international standards utilized in our road map, we recommend as follows:

- As regards to WMA DoH no. 16 to 18 and 33, we recommend a risk/benefit analysis according to the Evidence-based decision making adopted by United States (US) Department of Health and Human Services Agency for Healthcare Research and Quality (AHRQ) [see http://www.ahrq.gov (accessed at July 25, 2016)].

- To assess the adherence to CONSORT 2010 checklist, we recommend, as stated in the document: "we strongly recommend reading this statement [authors' note: Consort 2010 checklist] in conjunction with a CONSORT 2010 explanation and elaboration for important clarifications on all the items. If relevant, we also recommend reading CONSORT extensions for cluster randomized trials, noninferiority and equivalence trials, nonpharmacological treatments, herbal interventions, and pragmatic trials. Additional extensions are forthcoming: for those and for up-todate references relevant to this checklist [see http://www.consortstatement.org/consort-2010 (accessed July 25, 2016)].

- To assess the adherence to ICH/GCP no. 4.8 (informed consent), it will be required to comply with the checklist included in the same point of the document [http://ichgcp.net/48-informed-consent-oftrial-subjects (accessed July 25, 2016)].

\section{CONCLUSIONS}

It is necessary to ensure quality standards for surgery trials comparable with those for pharmacological trials. The use of a placebo and of a double-blind control group would improve the reliability of 
evidence, although the ethical assessment of surgery trials should be accurate and standardized both to prevent abuse or procedural bias, and, primarily, to ensure patient safety. We have thus proposed a "general sham surgery evaluation flowchart" as a possible practical tool that could be used to transfer to this specific field the three main international standards aimed at ensuring best practice in biomedical research: ICH/GCP Guidelines, the CONSORT checklist and the WMA-DoH, and also the new EU Regulation No 536/2014. ${ }^{11}$

This flowchart can be used at two levels: to provide the basic elements for surgical protocol design, which would represent the benchmark standard of care; and as a multiaxial assessment for considering the first two sources of the morality of human acts according to Aristotelian ethics: the object of the act (step 1) and some of its circumstances (steps 2-4). The consequences of the act are not assessed because the decision-making after the assessment should always be "pre-factum" and not "post-factum," as are the investigator's intentions, because they are an essential element for the ethical evaluation of a personal act, but not for the ethical assessment of a clinical case.

Further testing of the proposed flowchart is necessary, to evaluate both its scope and ease of use, and also its effectiveness in giving rise to suggested modifications to the general experimental design of a surgical trial.

\section{REFERENCES}

1. Merriam-Webster Medical Dictionary. Sham [Merriam-Webster website] 2015. Available at: http://www.merriam-webster.com/medical/sham. Accessed March 1, 2016.

2. Wartolowska K, Judge A, Hopewell S, et al. Use of placebo controls in the evaluation of surgery: systematic review. BMJ 2014 May 21 [348:g3253].
Available at: http://www.ncbi.nlm.nih.gov/pmc/articles/PMC4029190/pdf/ bmj.g3253.pdf. Accessed March 1, 2016.

3. Cobb LA, Thomas GI, Dillard DH, et al. An evaluation of internal mammary artery ligation by a double blind technic. N Engl J Med. 1959;260:1115-1158.

4. Beecher HK. Surgery as placebo. A quantitative study of bias. JAMA. 1961;176:1102-1107.

5. Horng S, Miller FG. Is placebo surgery ethical? N Engl J Med. 2002;347:137139.

6. Tenery R, Rakatansky H, Providence RI, et al. Surgical "placebo" controls. Ann Surg. 2002;235:303-307.

7. Royal College of Surgeons. Use of Placebo Surgery in Surgical Research [UK Royal College of Surgeons] Feb 2016. Available at: https://www.rcseng.ac.uk/ policy/documents/placebo-surgery-position-statement-february-2016.pdf. Accessed March 1, 2016.

8. Miller FG. Sham surgery: an ethical analysis. Am J Bioeth. 2003;3:41-48.

9. International Conference on Harmonisation of Technical Requirements for Registration of Pharmaceuticals for Human Use. ICH Harmonised Tripartite Guideline. Guideline for Good Clinical Practice E6 (R1) [International Conference on Harmonization web site] 1996. Available at: http://www.ich.org/fileadmin/Public_Web_Site/ICH_Products/Guidelines/Efficacy/E6/ E6_R1_Guideline.pdf. Accessed March 1, 2016.

10. World Medical Association. Declaration of Helsinki, Ethical Principles for Medical Research Involving Human Subjects [World Medical Association web site] 2013. Available at: http://www.wma.net/en/30publications/ 10policies/b3/. Accessed March 1, 2016.

11. EU Regulation No 536/2014 of the European Parliament and of the Council of 16 April 2014 on clinical trials on medicinal products for human use, and repealing Directive 2001/20/EC [European Union web site] 2014. Available at: http://eur-lex.europa.eu/legal-content/EN/TXT/PDF/?uri=CELEX:32014R0536\&from=it. Accessed March $1,2016$.

12. Schulz KF, Altman DGF, Moher D, for the Consort Group. CONSORT 2010 Statement: updated guidelines for reporting parallel group randomised trials. BMJ. 2010;340:c332. 